\title{
A Randomized Control Trial of Oral Sucrose Solution for Prevention of Hypoglycemia in High Risk Infants
}

\author{
SARIVIRIN SURACHAIDUNGTAVIL ${ }^{1}$, PITHI CHANVORACHOTE ${ }^{2}$ and NITHIPUN SUKSUMEK ${ }^{1}$ \\ ${ }^{1}$ Department of Pediatrics, Phramongkutklao Hospital and Phramongkutklao College of Medicine, Bangkok, Thailand; \\ ${ }^{2}$ Department of Pharmacology and Physiology, Faculty of Pharmaceutical Sciences, \\ Chulalongkorn University, Bangkok, Thailand
}

\begin{abstract}
Background: Neonatal hypoglycemia is found in up to $15 \%$ of neonates and $50 \%$ of those with risk factors. Hypoglycemia can cause brain damage and increase risk of developmental delay. Nevertheless, the data regarding hypoglycemia prevention by oral sucrose are still limited. The present study aimed to investigate whether oral sucrose solution can prevent hypoglycemia in high-risk infants. Patients and Methods: Four hundred and twenty-five infants with high hypoglycemic risk were randomized into two groups $(214$ infants in the intervention and 211 infants in the control groups). The intervention group received one dose of $0.8 \mathrm{ml} / \mathrm{kg}$ of $24 \%$ oral sucrose solution followed by enteral feed and was compared to the control group receiving enteral feed alone. Glucose levels were evaluated by Dextrostrix. Results: There was no significant difference in antenatal and perinatal risk factors of neonatal hypoglycemia between groups. Glucose level on admission was $72.1 \pm 20.3$ and $72.1 \pm 24.1 \mathrm{mg} / \mathrm{dl}$ in the intervention and control groups, respectively. Although no significant difference was recognized in terms of capillary blood glucose levels between groups, data analysis revealed that the glucose increase over time was significantly higher in the intervention group at $1 \mathrm{~h}$ (mean $\pm S E=3.61 \pm 1.27 \mathrm{mg} / \mathrm{dl}$; $p<0.005), 3 h(m e a n \pm S E=7.95 \pm 1.57 \mathrm{mg} / \mathrm{dl}, p<0.001)$, and 6 $h($ mean $\pm S E=6.31 \pm 1.62 \mathrm{mg} / \mathrm{dl}, p<0.001)$ in comparison to those of the control. No serious adverse event was observed in either group. Conclusion: A single dose of $24 \%$ sucrose
\end{abstract}

This article is freely accessible online.

Correspondence to: Dr. Nithipun Suksumek, Assistant Professor, Department of Pediatrics, Phramongkutklao Hospital and Phramongkutklao College of Medicine, Bangkok 10400, Thailand. Tel: +66 27634109, Fax: +66 23547827, e-mail: drnithipun@ hotmail.com; nithipun@gmail.com

Key Words: 24\% Oral sucrose solution, neonatal hypoglycemia, high-risk infants, early feeding. solution enhanced the increase of glucose level at 1, 3 and $6 \mathrm{~h}$. However, routine early feeding alone is not inferior to the addition of an oral sucrose solution.

A low glucose concentration in the blood of a newborn, i.e. neonatal hypoglycemia, is one of the most frequent abnormalities in the first period of life. Incidence of neonatal hypoglycemia is approximately $50 \%$ in high-risk newborns and $15 \%$ in healthy infants (1). The risk factors for hypoglycemia are prematurity, intrauterine growth restriction (IUGR), being large (LGA) or small (SGA) for gestational age, being an infant of a diabetic mother (IDM), hypothermia, asphyxia, polycythemia, and neonatal illnesses in various conditions, such as acute respiratory distress and sepsis (2). While the new data indicate that optimal treatment of hypoglycemic newborns is pressingly needed, the influencing factors, as well as a defined protocol are still lacking, and management varies according to the interpretations of individual physicians. Currently, there are several treatments including glucagon, glucocorticoids, dextrose solution, octreotide, and nifedipine which can be utilized (3). However, using such drugs in newborns is questionable in regard to long-term effects.

Interestingly, the use of oral sucrose is a common effective way to reduce distress and short-term pain during procedures in infants (4-8). Our previous study showed that an oral $24 \%$ sucrose solution significant relieved pain during examination for retinopathy of prematurity $(4,5,9,10)$. In addition, a $24 \%$ sucrose solution can be used to reduce pain from venipuncture (11). Although the use of oral sucrose administration is simple and widely accepted as a safe protocol, the evidence supporting the efficacy of such protocol on hypoglycemia in newborns is still limited.

The purpose of this study was to determine the effectiveness of $24 \%$ oral sucrose solution administration in conferring glucose homeostasis in the blood of high-risk neonates. Furthermore, the study investigated the response to sucrose solution among different sub-groups of infants having SGA, LGA, and IDMs. The information obtained 


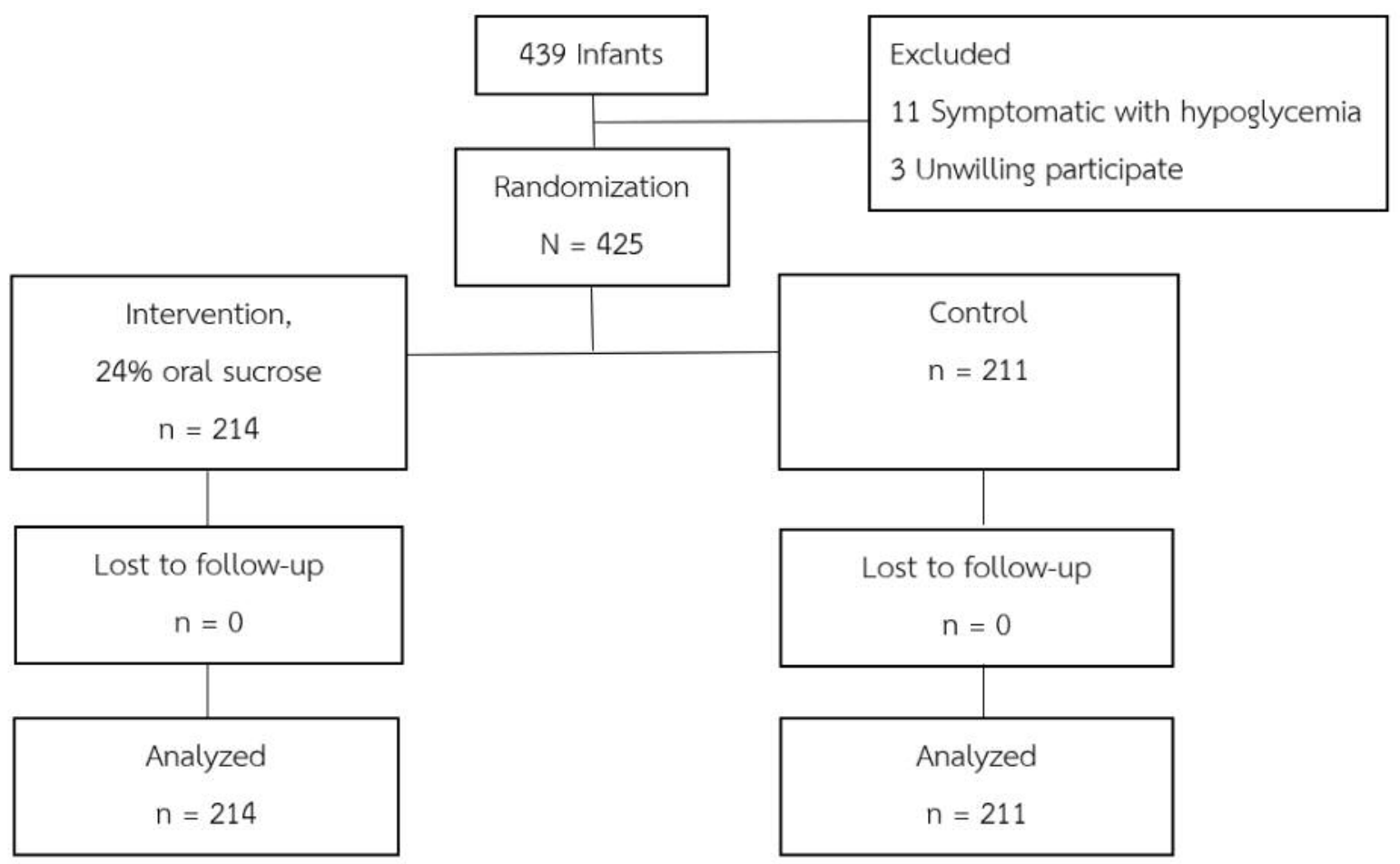

Figure 1. Consort diagram.

from the present study may be beneficial for ameliorating the complications of hypoglycemia in newborn infants and suggest a new safe and simple way of management.

\section{Patients and Methods}

This study was approved for ethical issues by the Institutional Review Board Royal Thai Army Medical Department (code $\mathrm{R} 046 \mathrm{~h} / 61$ ). The study was registered in Thai Clinical Trials Registry (code TCTR20190731002).

Study population. A randomized control trial was conducted during a 12-month period at the nursery unit and Neonatal Intensive Care Unit of Phramongkutklao Hospital. All infants at risk of neonatal hypoglycemia were recruited. The inclusion criteria were infants with gestational age at birth $\geq 35$ weeks with birth weight less than $2.5 \mathrm{~kg}$ or less than the $10^{\text {th }}$ percentile of their gestational age, or birth weight greater than $3.8 \mathrm{~kg}$ or more than the 90th percentile of their gestational age, and IDMs. Exclusion criteria included infants who had symptomatic hypoglycemia, congenital anomalies or dysmorphic features, congenital infections, and infants who were unable to receive oral sucrose solution or milk orally, due to $e$.g. gasping, vomiting or abdominal distension. Parents were given verbal and written information regarding the procedure. Patient data were collected and recorded in an electronic database using codes to maintain patient confidentiality and a security password was set to limit access to the data. The sample size estimation was based on the data from Harris et al.'s study (12). The calculated sample size was 203 infants per group, with power of $80 \%$ and significance level of 0.05 .
Data collection and measurement. Infants who met the selection criteria were randomized into the intervention and the control group by four randomization blocks provided in sequentially numbered opaque envelopes. Infants in the intervention group received $24 \%$ oral sucrose solution at $0.8 \mathrm{ml} / \mathrm{kg}$ (equal to $200 \mathrm{mg} / \mathrm{kg}$ ) in combination with breast milk or infant formula. Those in the control group received only milk. The infants were assessed by a neonatologist or senior pediatric resident. They were monitored for heart rate and oxygen saturation by Masimo SET Radical Signal Extraction PulseOximeter (Irvine, CA, USA) before, during and after the oral sucrose or milk administration. The initial sugar level of capillary blood by Dextrostix (Ames Co., Stoke Poges, UK) in both groups was evaluated within $15 \mathrm{~min}$ of life or as soon as possible when high-risk infants were transferred to the nursery unit. The intervention group received $24 \%$ oral sucrose solution by slow drip through a syringe into the cheek bulge within 1 minute. In the case of vomiting or suffocating, the solution would be stopped and those infants would be withdrawn from the study. The data collection process consisted of the sugar level at the age of $0 \mathrm{~h}, 30 \mathrm{~min}, 1 \mathrm{~h}, 3$ $\mathrm{h}$, and $6 \mathrm{~h}$. Admission to the nursery or NICU, complications of hypoglycemia such as drowsiness, seizures, difficulty breathing, apnea, receiving ventilator ventilation, length of hospitalization stayed, neonatal and perinatal mortality rate were documented.

Statistical analysis. Descriptive statistics are presented as the mean and SE for continuous variables, and count and percentage for categorical data. Quantitative variables were compared between groups using the Mann-Whitney $U$-test. Continuous data comparison using unpaired $t$-test and repeated measures ANOVA. 
Table I. Basic characteristics of infants in the group that had received $24 \%$ oral sucrose solution (intervention) and the control group.

\begin{tabular}{lccc}
\hline & $\begin{array}{c}\text { Intervention } \\
(\mathrm{n}=214)\end{array}$ & $\begin{array}{c}\text { Control } \\
(\mathrm{n}=211)\end{array}$ & $p$-Value \\
\hline Gestational age (weeks) & $37.6 \pm 1.4$ & $37.6 \pm 1.5$ & 0.98 \\
Birthweight (g) & $2996 \pm 637.3$ & $3067 \pm 648.5$ & 0.56 \\
Male (n, \%) & $102(48.3)$ & $100(46.7)$ & 0.74 \\
Apgar score at 1 min & $8 \pm 0.7$ & $8 \pm 0.5$ & 0.38 \\
(mean \pm SD) & & & \\
Apgar score at 5 min & $9 \pm 0.3$ & $9 \pm 0.2$ & 0.57 \\
Size for age & & & 0.45 \\
$\quad$ AGA (n, \%) & $101(47.9)$ & $92(43)$ & \\
SGA (n, \%) & $64(30.3)$ & $77(36)$ & \\
$\quad$ LGA (n, \%) & $46(21.8)$ & $45(21)$ & \\
Maternal factor (n) & & & \\
$\quad$ Gestational diabetes (n, \%) & $86(40.8)$ & $84(39.3)$ & 0.75 \\
$\quad$ Pre-eclampsia (n, \%) & $21(10)$ & $30(14)$ & 0.20 \\
Prolonged rupture of & $18(8.5)$ & $12(5.6)$ & 0.24 \\
$\quad$ membrane (n, \%) & & & \\
Antenatal steroids (n, \%) & $7(3.3)$ & $8(3.7)$ & 0.81 \\
Time to start feeding (min) & $12.9 \pm 6.1$ & $13.4 \pm 5.8$ & 0.36 \\
Volume of feeding (ml) & $15.7 \pm 5$ & $15.8 \pm 5$ & 0.98 \\
\hline
\end{tabular}

AGA: Appropriate for gestational age; SGA: small for gestational age; LGA: large for gestational age.

The categorical variables were evaluated using chi-square test. Statistical analysis was carried out using SPSS Statistical Software (SPSS version 22; IBM, New York, NY, USA). p-Values of less than 0.05 were considered statistically significant.

\section{Results}

General and baseline characteristics of enrolled infants. Four hundred and thirty-nine neonates met the eligibility criteria for determining high-risk for neonatal hypoglycemia. Fourteen infants were excluded, three due to lack of parental consent and 11 who presented with symptomatic hypoglycemia prior to being admitted to the nursery. Four hundred and twentyfive high-risk infants were randomly assigned into two groups: 214 were assigned to receive $24 \%$ oral sucrose solution with routine early breast milk or formula feeding, and 211 infants were the control group, receiving routine early breast milk or formula feeding only. All infants who participated in the study were followed-up until the end of the study, as shown in CONSORT DIAGRAM (Figure 1).

There was no significant difference in gestational age and birth weight for the intervention and the control group (Table I). Baseline characteristic included size proportion. There were no differences in maternal conditions, including diabetes in mothers, hypertension during pregnancy, chorioamnionitis, and antenatal steroid prophylaxis. Both groups received early feeding (breast milk or formula) immediately after transferal to the nursery and there were no differences in the amount of feeding as shown in Table I.
Table II. Glucose level at indicated time points.

\begin{tabular}{lcccc}
\hline \multirow{4}{*}{ Time point } & \multicolumn{2}{c}{ Glucose level, mg/dl } & & \\
\cline { 2 - 4 } & $\begin{array}{c}\text { Intervention } \\
(\mathrm{n}=214)\end{array}$ & $\begin{array}{c}\text { Control } \\
(\mathrm{n}=211)\end{array}$ & $p$-Value* & $p$-Value** \\
\hline At birth & $70.1 \pm 20$ & $72.1 \pm 24$ & 0.360 & 0.51 \\
30 min & $69.8 \pm 19$ & $69.7 \pm 23$ & 0.983 & \\
$1 \mathrm{~h}$ & $73.7 \pm 15$ & $75.0 \pm 18$ & 0.423 & \\
$3 \mathrm{~h}$ & $78.0 \pm 16$ & $78.5 \pm 15$ & 0.740 & \\
$6 \mathrm{~h}$ & $76.4 \pm 14$ & $76.7 \pm 15$ & 0.799 & \\
\hline
\end{tabular}

*Unpaired $t$-test. **Repeated measures ANOVA.

Increase of capillary blood glucose level over time. We monitored the time-dependent increase of capillary blood glucose using Dextrostix as described in the Patients and Methods. Results showed that at baseline, the glucose levels in both groups were comparable (Table II). At $30 \mathrm{~min}, 1 \mathrm{~h}$, $3 \mathrm{~h}$, and $6 \mathrm{~h}$ after birth, there were no significant differences in glucose level between intervention and control groups, as shown in Table II. Comparative study of glucose increases further revealed that the increase in glucose at $30 \mathrm{~min}$ over that at baseline was not significantly different between groups (Table III). Interestingly, the increase of glucose was notably high in infants with birthweight less than $2.5 \mathrm{~kg}$ (Table IV). Moreover, infants with birthweight less than 2.5 $\mathrm{kg}$ from diabetic mothers were found to be more sensitive to the intervention, indicated by the significant increase in glucose at 1 , and $6 \mathrm{~h}$, as shown in Table $\mathrm{V}$.

Observation of adverse events. There was no death observed during the study. There were no differences in the rate of NICU admission, intravenous glucose administration, or length of hospital stay $(2.4 \%$ and $1.4 \%, 7.6 \%$ and $10.7 \%$, and $3.7 \pm 3$ and $3.8 \pm 2$ days for the intervention and control groups, respectively)

\section{Discussion}

Glucose is a critical component in providing energy for brain metabolism and growth of the fetus. There is an increasing evidence of neonatal hypoglycemia, which may have longterm neurological outcomes (13), especially in providing energy to brain cells. Hypoglycemia in infants will increase the risk of poor development and problematic behavior of the children in the future. Most cases of neonatal hypoglycemia are transient, respond readily to treatment, and are associated with an excellent prognosis (14). Concerning neonatal hypoglycemia in high-risk infants, there are several guidelines for management to prevent this condition, including guidelines from the American Academy of Pediatrics (AAP) (12) for reference in tracking glucose 
Table III. Comparison of change in glucose level at each time point relative to baseline at birth within groups.

\begin{tabular}{|c|c|c|c|c|c|c|}
\hline \multirow{3}{*}{$\begin{array}{l}\text { Time from } \\
\text { birth }\end{array}$} & \multicolumn{6}{|c|}{ Change in glucose level, $\mathrm{mg} / \mathrm{dl}$} \\
\hline & \multicolumn{3}{|c|}{ Intervention $(\mathrm{n}=214)$} & \multicolumn{3}{|c|}{ Control $(n=211)$} \\
\hline & Mean & SE & $p$-Value & Mean & SE & $p$-Value \\
\hline $30 \mathrm{~min}$ & -0.289 & 1.039 & 0.781 & -2.319 & 1.218 & 0.058 \\
\hline $1 \mathrm{~h}$ & 3.611 & 1.275 & $<0.005^{*}$ & 2.995 & 1.389 & $0.032 *$ \\
\hline $3 \mathrm{~h}$ & 7.948 & 1.57 & $<0.001 *$ & 6.451 & 1.821 & $<0.001 *$ \\
\hline $6 \mathrm{~h}$ & 6.308 & 1.622 & $<0.001 *$ & 4.657 & 1.748 & $0.008^{*}$ \\
\hline
\end{tabular}

*Unpaired t-test..

levels. The aforementioned approach of the AAP also monitors the risk of neonatal hypoglycemia. This approach provides immediate management for neonatal hypoglycemia in order to prevent further morbidity. The treatment requires infant patients to be admitted to the hospital with early feeding or intravenous dextrose administration. This may result in the separation of a mother and baby, leading to the reduction of the relationship between them after birth. In addition, it may cause prolonged hospitalization.

The purpose of this study was to evaluate blood glucose levels in infants at risk of hypoglycemia, including low birth weight infants ( $<2.5 \mathrm{~kg}$ or $<10^{\text {th }}$ percentile), overweight infants $\left(>3.8 \mathrm{~kg}\right.$ or $>90^{\text {th }}$ percentile) and IDMs. Two hundred and fourteen were assigned to receive $24 \%$ oral sucrose solution with routine early breast milk or formula feeding and 211 infants comprised the control group, receiving only routine early breast milk or formula feeding. All infants who participated in the study were followed-up until the end of the study.

This study found that there were significant differences in glucose levels in the subgroup comparison. In the intervention group receiving oral sucrose solution and early feeding, the glucose level increased 3.6, 7.9, and $6.3 \mathrm{mg} / \mathrm{dl}$ from baseline at 1,3 , and $6 \mathrm{~h}$ of life, respectively, whereas it only significantly increased at $3 \mathrm{~h}$ of life in the control group. Therefore, from a clinical perspective, $24 \%$ oral sucrose administration may reduce the clinical manifestation of hypoglycemia and may be a safe routine monitoring. Harris $e t$ al. (12) and Hegarty et al. (15) studied the applicability of $40 \%$ oral glucose gel administration in infants at risk of hypoglycemia, such as IDM, late preterm infants, SGA and LGA, compared to placebo administration. They found that $40 \%$ oral glucose gel reduced the rate of neonatal hypoglycemia and the rate of NICU admission. However, oral glucose gel is not currently imported into Thailand; instead, the administration of $24 \%$ oral sucrose solution can be used as demonstrated in our study. This is also cheaper and easy to prepare because it can be manufactured in a hospital in the public health system of Thailand.
Table IV. Comparison of changes in glucose level between groups stratified by birthweight. Data are means \pm standard error.

\begin{tabular}{lcccc}
\hline & & \multicolumn{2}{c}{ Change in glucose level, mg/dl } & \\
\cline { 3 - 4 } Birthweight, & $\begin{array}{c}\text { Time from } \\
\text { birth }\end{array}$ & $\begin{array}{c}\text { Intervention } \\
(\mathrm{n}=214)\end{array}$ & $\begin{array}{c}\text { Control } \\
(\mathrm{n}=211)\end{array}$ & \\
\hline$<2.5$ & $30 \mathrm{~min}$ & $2.26 \pm 14.9$ & $-5.40 \pm 18.69$ & $0.017^{*}$ \\
& $1 \mathrm{~h}$ & $5.76 \pm 18.28$ & $1.00 \pm 22.93$ & 0.222 \\
& $3 \mathrm{~h}$ & $12.83 \pm 23.55$ & $3.00 \pm 28.50$ & $0.046^{*}$ \\
& $6 \mathrm{~h}$ & $12.65 \pm 26.9$ & $2.33 \pm 28.45$ & $0.047^{*}$ \\
$2.5-3.8$ & $30 \mathrm{~min}$ & $-1.22 \pm 16.03$ & $-1.46 \pm 17.54$ & 0.911 \\
& $1 \mathrm{~h}$ & $2.58 \pm 19.87$ & $3.23 \pm 18.40$ & 0.788 \\
& $3 \mathrm{~h}$ & $6.82 \pm 23.43$ & $7.74 \pm 25.16$ & 0.761 \\
$>3.8$ & $6 \mathrm{~h}$ & $4.00 \pm 22.89$ & $6.17 \pm 23.42$ & 0.456 \\
& $30 \mathrm{~min}$ & $-0.89 \pm 9.64$ & $0.93 \pm 15.85$ & 0.614 \\
& $1 \mathrm{~h}$ & $4.3 \pm 10.69$ & $6.33 \pm 21.79$ & 0.665 \\
& $3 \mathrm{~h}$ & $3.63 \pm 16.49$ & $8.78 \pm 28.06$ & 0.415 \\
& $6 \mathrm{~h}$ & $4.74 \pm 17.19$ & $3.08 \pm 27.98$ & 0.794 \\
\hline
\end{tabular}

*Unpaired $t$-test.

Table V. Comparison of difference of glucose level between group in infants with birthweight less than $2.5 \mathrm{~kg}$ born to diabetic mothers.

Change in glucose level, $\mathrm{mg} / \mathrm{dl}$

\begin{tabular}{lccl}
\cline { 2 - 3 } Time from birth & Intervention $(4 / 53)$ & Control $(\mathrm{n}=9 / 64)$ & $p$-Value \\
\hline $30 \mathrm{~min}$ & $15.5 \pm 17.82$ & $-13.78 \pm 30.63$ & 0.107 \\
$1 \mathrm{~h}$ & $23 \pm 18.02$ & $-4.78 \pm 18.55$ & $0.029^{*}$ \\
$3 \mathrm{~h}$ & $20.5 \pm 19.09$ & $-6.44 \pm 24.68$ & 0.080 \\
$6 \mathrm{~h}$ & $32.75 \pm 19.22$ & $-7.67 \pm 21.07$ & $0.007^{*}$ \\
\hline
\end{tabular}

*Unpaired $t$-test.

There were no differences between groups in the glucose level at each time point (30 min, 1, 3, $6 \mathrm{~h}$ of life) comparing between control routine feeding and oral sucrose administration. This study revealed that early feeding alone was not inferior to early feeding with adjuvant oral sucrose solution. We therefore recommend that early feeding (breast milk or formula feeding) is appropriate clinical care for highrisk babies. Moreover, advantages of early feeding may include the promotion of breast milk production, improve breastfeeding, and reduce hospital cost as described in Harding et al.'s study (1).

However, we did find that administration of oral sucrose solution in infants with birthweight lower than $2.5 \mathrm{~kg}$ significantly increased the blood glucose level at $30 \mathrm{~min}, 3 \mathrm{~h}$, and $6 \mathrm{~h}$ after birth comparing to the control groups. Consistently, oral sucrose significantly increased the blood glucose of IDMs with birthweight lower than $2.5 \mathrm{~kg}$ at 1 and 6 $\mathrm{h}$ after birth compared to the normal feeding control. Therefore, 
such administration of oral sucrose may be beneficial for the management of high-risk infants in order to rapidly increase the blood glucose level. There will be a need to monitor for symptoms for a longer period to determine whether there is a clinically significant difference, although short-term results including need for intravenous glucose, NICU admission, and length of hospital stay were not difference and there were no deaths in either group. The advantage of this study is that it was a randomized control trial with a large number of participants. We provided a proper tracking of sugar levels in each period and all infants were monitored for data collection. Importantly, all infants still received standard treatment according to AAP guidelines $(2,7,8)$.

\section{Conflicts of Interest}

The Authors declare that there are no conflicts of interest in regard to this research.

\section{Authors' Contributions}

SS collected the data. NS designed and performed the experiments. NS and PC analyzed the data. NS wrote the article. All Authors approved the final article.

\section{Acknowledgements}

This work was supported by grants from Phramongkutklao Hospital and Phramongkutklao College of Medicine, Royal Thai Army Medical Department.

\section{References}

1 Harding JE, Hegarty JE, Crowther CA, Edlin R, Gamble G and Alsweiler JM: Randomized trial of neonatal hypoglycaemia prevention with oral dextrose gel (hPOD): study protocol. BMC Pediatr 15: 120, 2015. PMID: 26377909. DOI: 10.1186/s12887015-0440-6

2 Committee on Fetus and Newborn: Postnatal glucose homeostasis in late-preterm and term infants. Pediatrics 127(3): 575-579, 2011. PMID: 21357346. DOI: 10.1542/peds.2010-3851

3 Adamkin DH: Neonatal hypoglycemia. Curr Opin Pediatr 28(2): 150-155, 2016. PMID: 26780301. DOI: 10.1097/MOP 0000000000000319

4 Gal P, Kissling GE, Young WO, Dunaway KK, Marsh VA, Jones SM, Shockley DH, Weaver NL, Carlos RQ and Ransom JL: Efficacy of sucrose to reduce pain in premature infants during eye examinations for retinopathy of prematurity. Ann Pharmocother 39: 1029-1033, 2005. PMID: 15855243. DOI: 10.1345/aph.1E477

5 Mitchell A, Stevens B, Mungan N, Johnson W, Lobert S and Boss B: Analgesic effects of oral sucrose and pacifier during eye examinations for retinopathy of prematurity. Pain Manag Nurs 5(4): 160-168, 2004. PMID: 15616486. DOI: 10.1016/j.pmn.2004.06.001
6 Boyle EM, Freer Y, Khan-Orakzai Z, Watkinson M, Wright E, Ainsworth JR and McIntosh N: Sucrose and non-nutritive sucking for the relief of pain screening for retinopathy of prematurity: A randomized controlled trial. Arch Dis Child Fetal Neonatal Ed 91(3): F166-168, 2006. PMID: 16428355. DOI: 10.1136/adc.2005.087668

7 Rush R, Rush S, Ighani F, Anderson B, Irwin M and Naqvi M: The effects of comfort care on the pain response in preterm infants undergoing screening for retinopathy of prematurity. Retina 25: 59-62, 2005. PMID: 15655442. DOI: 10.1097/ 00006982-200501000-00008

8 Suksumek N, Jumroendararasame C, Thitiratsanont N, Sutarapanakit N and Punnahitananda S: A randomized, doubleblind trial of oral sucrose solution and placebo for pain relief in retinopathy of prematurity examination. J Med Assoc Thai 100 (5): 521, 2017

9 Costa MC, Eckert GU, Fortes BG, Fortes Filho JB, Silveira RC and Procianoy RS: Oral glucose for pain relief during examination for retinopathy of prematurity: A masked randomized clinical trial. Clinics (Sao Paulo) 68(2): 199-204, 2013. PMID: 23525316. DOI: 10.6061/clinics/2013(02) oa13

10 O'Sullivan A, O'Connor M, Brosnahan D, McCreery K and Dempsey EM: Sweeten, soother and swaddle for retinopathy of prematurity screening: a randomised placebo-controlled trial. Arch Dis Child Fetal Neonatal Ed 95(6): F419-422, 2010. PMID: 20876596. DOI: 10.1136/adc.2009.180943

11 De Bernardo G, Riccitelli M, Sordino D, Giordano M, Piccolo S, Buonocore G and Perrone S: Oral 24\% sucrose associated with nonnutritive sucking for pain control in healthy term newborns receiving venipuncture beyond the first week of life. J Pain Res 12: 299-305, 2019. PMID: 30662283. DOI: 10.2147/ JPR.S184504

12 Harris DL, Weston PJ, Signal M, Chase JG and Harding JE: Dextrose gel for neonatal hypoglycaemia (the Sugar Babies Study): A randomised, double-blind, placebo-controlled trial. Lancet 382: 2077-2083, 2013. PMID: 24075361. DOI: 10.1016/ S0140-6736(13)61645-1

13 Cowett RM and Loughead JL: Neonatal glucose metabolism: differential diagnoses, evaluation, and treatment of hypoglycemia. Neonatal Netw 21(4): 9-19, 2002. PMID: 12078323. DOI: $10.1891 / 0730-0832.21 .4 .9$

14 Kallem VR, Pandita A and Gupta G: Hypoglycemia: When to treat? Clin Med Insights Pediatr 11: 1179556517748913, 2017. PMID: 29276423. DOI: 10.1177/1179556517748913

15 Hegarty JE, Harding JE, Gamble GD, Crowther CA, Edlin R and Alsweiler JM: Prophylactic oral dextrose gel for newborn babies at risk of neonatal hypoglycaemia: a randomised controlled dose-finding trial (the Pre-hPOD Study). PLoS Med 13(10): e1002155, 2016. PMID: 27780197. DOI: 10.1371/journal. pmed.1002155
Received February 13, 2020

Revised March 2, 2020

Accepted March 5, 2020 